\title{
Cansancio y somnolencia en conductores de ómnibus y accidentes de carretera en el Perú: estudio cuantitativo
}

\author{
Jorge Rey de Castro, ${ }^{1}$ Jorge Gallo ${ }^{2}$ y Hugo Loureiro ${ }^{2}$
}

Forma de citar

Rey de Castro J, Gallo J, Loureiro H. Cansancio y somnolencia en conductores de ómnibus y accidentes de carretera en el Perú: estudio cuantitativo. Rev Panam Salud Publica. 2004;16(1):11-8.

RESUMEN Objetivo. Evaluar la relación del cansancio y la somnolencia en los conductores de ómnibus con los accidentes de carretera. La información obtenida de diversos países atribuye a la somnolencia del conductor una parte importante en la génesis de los accidentes de carretera. No obstante, la información al respecto es escasa en el caso del Perú.

Métodos. Se realizó un estudio transversal, de índole observacional y comparativa, basado en una encuesta prevalidada y de aplicación supervisada, entre 238 conductores de ómnibus que circulan por la carretera Panamericana Norte del Perú. Para determinar la relación entre variables se emplearon la prueba de ji al cuadrado y el indice de Pearson, con un valor de significación de $\mathrm{P}<0,05$. Variables de análisis: cansancio, somnolencia, horas de conducción por dí, horas de sueño por día, indice de masa corporal, ronquido, pausas respiratorias y el antecedente de haber tenido o estar a punto de tener un accidente durante la conducción.

Resultados. De los 238 conductores, todos ellos varones, 45\% refirieron haber estado a punto de tener un accidente o haberlo sufrido durante la conducción, 55\% dormian menos de 6 horas al día, 31\% habian dormido menos de 6 horas en las últimas 24, y 80\% acostumbraban conducir más de 5 horas sin descanso. Señalaron cansancio durante la conducción $56 \%$ y, de estos, $65 \%$ lo experimentaban durante la madrugada. Setenta y seis (32\%) conductores reconocieron que pestañeaban durante la conducción. Ciento noventa y cuatro (81\%) siempre dormían en el maletero, estuviera el ómnibus en ruta o en la terminal. Maniobras para evitar dormirse: mojarse la cara con agua, comer fruta, abrir ventana de la cabina, beber café, escuchar música, fumar, masticar (chacchar) coca y beber alcohol con hoja de coca. En opinión de $55 \%$ de los conductores, la primera causa de accidentes de carretera es el cansancio. Los accidentes durante la conducción, virtuales o consumados, ocurrieron predominantemente entre las 00:00 y las 06:00 horas. Este antecedente mostró firme asociación $(\mathrm{P}<0,0005)$ con el pestañeo y el cansancio.

Conclusiones. La somnolencia y el cansancio durante la conducción fueron frecuentes. Su origen puede ser multifactorial: privación aguda y crónica del sueño, rotación desordenada de horarios y trastornos del sueño de origen ambiental. Los resultados respaldan la hipótesis de que hay una relación entre la fatiga y somnolencia de los conductores y los accidentes en carreteras.

Palabras clave Accidentes de tránsito, Perú.

1 Universidad Peruana Cayetano Heredia, Facultad de Medicina, Clínica Anglo-Americana, Lima, Perú. Toda correspondencia deberá dirigirse a: Jorge Rey de Castro, Av. Alfredo Salazar 314, Ofi-
2 Universidad Peruana Cayetano Heredia, Facultad de Medicina, Lima, Perú. 
Dormir es una necesidad neurobiológica que alterna en un patrón más o menos predecible con estados de vigilia. Se han señalado más de 80 factores que producen alteraciones del sueño, de la vigilia o de ambos (1). La somnolencia deteriora variables funcionales psicomotoras y neurocognoscitivas, como el tiempo de reacción, la capacidad de vigilancia, juicio y atención, así como el procesamiento de información, lo cual puede significar accidentes de tránsito para los conductores de vehículos o accidentes de trabajo para los operadores de maquinarias potencialmente peligrosas (2). En la situación extrema el conductor puede pestañear, cabecear y finalmente dormirse durante la conducción. En cualquier caso, la somnolencia determina una pérdida de la capacidad de efectuar maniobras evasivas para evitar estos percances, que suelen acarrear altas tasas de morbimortalidad por evento y grandes pérdidas en infraestructura derivadas de la destrucción de los vehículos y de otros daños materiales (3).

El objetivo del presente estudio es evaluar la asociación entre el cansancio y somnolencia de los conductores y los accidentes de tránsito en las carreteras del Perú, además de destacar la importancia de estos factores. Información originada en distintos países es enfática en señalar una clara relación entre la somnolencia en los conductores y el riesgo de accidentes en zonas urbanas y carreteras. De acuerdo con esas fuentes, la somnolencia ocasiona accidentes con una frecuencia que fluctúa entre 16 y 23\% de los casos (2, 4-6). La literatura médica relacionada con el tema proviene casi exclusivamente de Europa y América del Norte. En lo que se refiere al Perú, no se cuenta con información objetiva que permita conocer las variables fundamentales que inciden en el problema. Una revisión sistemática de los accidentes de ómnibus ocurridos en las carreteras peruanas y publicados en el diario El Comercio entre los años 1999 y 2000 documentó, en la perspectiva periodística, que este tipo de siniestros estaría vinculado a altas tasas de morbilidad y mortalidad (7). El presente informe ingresa en un terreno, hasta hoy inexplorado en este país, que reviste interés médico y que atañe desde luego a la salud pública.

\section{MATERIALES Y MÉTODOS}

El estudio se desarrolló en la terminal terrestre Fiori, ubicada en el kilómetro 10 de la Carretera Panamericana Norte (distrito de San Martín de Porres), en la zona norte de la Lima metropolitana, durante marzo de 2002. Esta terminal es paradero principal y obligatorio de ómnibus que viajan por toda la costa y la sierra norte del país.

Participaron 238 conductores de un total de aproximadamente 400 que se calcula operan en la terminal según indagaciones del propio estudio, ya que no hay registros oficiales que ofrezcan una cifra exacta. Un análisis cualitativo de esta misma población, realizado en el año 2001 (8), sirvió de pauta para el diseño y la puesta en marcha de la presente investigación.

El estudio, de diseño transversal, se propuso ser, por un lado, descriptivo de los hábitos de los encuestados $\mathrm{y}$, por el otro, comparativo entre las características de los conductores que refieren (grupo A) y no (grupo B) el antecedente de haberse accidentado o casi accidentado durante la conducción de sus unidades.

Se aplicó un cuestionario supervisado que en su versión final contenía 23 preguntas. El diseño de este instrumento se basó en buena parte en preguntas extraídas de los cuestionarios de McCartt et al. (9), de la National Sleep Foundation $(10,11)$ y de un cuestionario clínico empleado en nuestro centro, validado previamente por el grupo de los autores (12). La información obtenida del estudio cualitativo mencionado en párrafos anteriores permitió emplear terminología propia del vocabulario de los conductores (8).

El cuestionario fue sometido a una prueba piloto de validación un mes antes del estudio en 30 conductores de la misma terminal, en un proceso que permitió diseñar el instrumento definitivo. Se obtuvieron datos referentes a edad, sexo, talla, peso, hábitos laborales y de sueño, así como el antecedente o no de haber tenido un accidente inminente o consumado. Las preguntas fueron de tipo cerrado, con respuesta afirmativa o negativa en unos casos, y escala cuantitativa ("nunca", "rara vez", "alguna vez", "muchas veces" y "siempre") en otros; solo una pregunta fue de respuesta abierta: “¿Cuál cree usted que es la causa más importante de los accidentes de carretera?".

Para efectos del estudio se impartió capacitación a cinco encuestadores con experiencia en trabajo de campo, uno de los cuales había participado en la prueba piloto. La encuesta se aplicó en el área de la terminal donde se concentran las unidades de las varias empresas de transporte y donde los conductores hacen parada obligatoria. Se midieron la estatura y el peso de todos los encuestados con instrumentos previamente calibrados. Los participantes no recibieron aliciente alguno por su participación y al finalizar la entrevista se les entregó un folleto de resumen con recomendaciones específicas sobre buenos hábitos de sueño para los conductores. El período de recopilación de datos abarcó cuatro días, lo cual, aunado a la constante rotación de conductores en la terminal, debió reducir la oportunidad de que los participantes intercambiaran opiniones relacionadas con el contenido de la encuesta.

El entorno laboral. Las condiciones de trabajo de estos conductores, tanto en la terminal como a lo largo de la ruta, distan mucho de lo que suelen referir los estudios sobre conductores que laboran en Europa o América del Norte. Por ello parece conveniente describirlas brevemente. En muchos casos, los horarios de partida de las unidades están supeditados a completar los lugares vacantes. Cada conductor puede trabajar en horario diurno, vespertino o nocturno, sin sistemática de reposo previo o ulterior. Con frecuencia dos conductores alternan la responsabilidad del volante a lo largo de la ruta y el que descansa lo hace en el maletero (portaequipaje), situado en la parte inferior de la unidad. Cuando el ómnibus se detiene en su paradero final, el conductor se encarga de supervisar la 
entrega del equipaje, limpiar la unidad y realizar labores de mantenimiento y a veces de mecánica; lo cual reduce ostensiblemente el período real de reposo. Ni la terminal Fiori ni los paraderos a lo largo de la ruta disponen de áreas adecuadas para el descanso de los conductores, por lo cual la práctica sistemática de reposar en el maletero se cumple incluso cuando el ómnibus se encuentra estacionado, en medio del bullicio originado por los pasajeros, el ruido de otras unidades y la alta temperatura ambiental que distingue a la zona norte del país. Los conductores de ómnibus trabajan a destajo, en general carecen de prestaciones (beneficios) sociales y en la mayor parte de los casos visitan a su familia una vez por semana. La información sobre estos aspectos se obtuvo en el estudio cualitativo previo (8).

Esta investigación formó parte de la actividad académica de uno de los autores (JG) para obtener el Grado de Bachiller en Medicina de la Universidad Peruana Cayetano Heredia. En ese carácter, el proyecto fue presentado y aceptado por las instancias correspondientes del Departamento de Medicina de la Facultad de Medicina Alberto Hurtado de esa institución universitaria. En el orden ético, los conductores autorizaron su participación en el estudio al acceder a la encuesta.

Definiciones. En la práctica clínica es frecuente que, en la perspectiva del paciente, los términos somnolencia, cansancio y fatiga se superpongan o se utilicen como sinónimos. La investigación cualitativa por medio de la técnica de grupos focales (8) y la prueba piloto basada en el cuestionario permitieron identificar la connotación de estos términos en la población de estudio. Los conductores de la terminal Fiori utilizaron la palabra "cansancio" para expresar sensación de falta de energía, pesadez de cabeza o cuello, cefalea, modorra y disminución de la capacidad de atención o concentración. En cambio, respondieron invariablemente de forma negativa al ser interrogados por "somnolencia" o "quedarse dormido" durante la conducción. De ahí que en la encuesta se optara por emplear el término "cansancio".

Todos los datos se registraron en un archivo de computadora con el programa Excel y sometidos a análisis estadístico con el paquete SPSS, versión 10.0. Se emplearon mediciones de tendencia central, como la media aritmética, y de dispersión, como la desviación estándar. Se usó la prueba de ji al cuadrado para evaluar la relación entre variables y se calculó el índice de correlación de Pearson; se consideró estadísticamente significativa una diferencia con un valor $P \leq 0,05$.

\section{RESULTADOS}

El total de 238 participantes representa $59 \%$ de la población total estimada de choferes de la terminal Fiori; $4 \%$ se negaron a participar. Las características de la población de conductores, todos varones, se resumen en el cuadro 1. La edad promedio de toda la población encuestada fue 42.5, con límites de 19 y 73 años. Solo la variable de horas de conducción por día mostró diferencia estadísticamente significativa entre el grupo A $(n=108)$ y el B $(n=130)$.

Respecto al número de horas que los conductores suelen dormir al día, $47 \%$ lo hacen menos de 7 horas y $40 \%$ menos de 6; no hubo diferencia al respecto entre los grupos A y B. Al momento de la encuesta, 31\% conductores habían dormido menos de 6 horas de las últimas 24. Tampoco hubo diferencia entre los grupos en esta variable. Hasta $80 \%$ de los conductores suelen conducir más de 5 horas sin detenerse.
La frecuencia referida de cansancio durante la conducción, una variable que abarcó desde "alguna vez" hasta "siempre", fue de 56\%. La percepción de cansancio se ubicó predominantemente en las horas de la madrugada en $65 \%$ y en las de la tarde en $20 \%$. Del total, $32 \%$ reconocieron haber pestañeado mientras conducían.

Una proporción de $81 \%$ de los encuestados afirmó que "siempre" dormía en el maletero del ómnibus y $8 \%$ que lo hacía desde "muchas veces" hasta "rara vez". La mitad dormían mientras el ómnibus estaba en ruta, es decir, cuando su compañero los reemplazaba al volante, $4 \%$ solo en la terminal cuando la unidad se encontraba estacionada y $46 \%$ en ambas circunstancias.

Los conductores especificaron una variedad de maniobras para evitar quedarse dormidos durante la conducción (87\% empleaban por lo menos una maniobra), como son: mojarse la cara $(27 \%)$, comer fruta $(18 \%)$, abrir la ventana para aumentar la ventilación de la cabina $(14 \%)$, beber café $(14 \%)$, escuchar música (6\%), fumar (4\%), masticar (cacchar) coca (4\%) y beber una mezcla de alcohol con hoja de coca $(2 \%)$.

En cuanto a patrones del sueño, 37\% de los conductores declararon roncar y $1,2 \%$ indicaron pausas respiratorias durante el dormir, según referencia de personas allegadas que podían advertir estos signos. Por lo demás, 35\% refirieron haber experimentado, por lo menos alguna vez, cansancio durante la conducción de sus unidades.

En la opinión de los encuestados, las causas más frecuentes de accidentes en las carreteras del Perú son: cansancio

CUADRO 1. Características de la población de conductores de ómnibus, según el antecedente positivo (Grupo A) o negativo (Grupo B) de accidente inminente o consumado. Lima, Perú

\begin{tabular}{lccl}
\hline & Grupo A $(n=108)$ & Grupo B $(n=130)$ & $P$ \\
\hline Edad (en años) & $42,7 \pm 10.1^{\text {a }}$ & $42,2 \pm 9,9$ & 0,75 \\
Índice de masa corporal & $29 \pm 4,4$ & $29,6 \pm 3,9$ & 0,29 \\
Años de ser chofer & $17,3 \pm 9,8$ & $17 \pm 10,3$ & 0,8 \\
Horas de conducción al día & $7,9 \pm 2,5$ & $6,9 \pm 2,4$ & 0,003 \\
Horas de sueño al día & $7,1 \pm 1,9$ & $7,5 \pm 2,4$ & 0,1 \\
\hline
\end{tabular}

${ }^{a}$ Media \pm desviación estándar. 
FIGURA 1. Número de accidentes inminentes o consumados, según la hora del día, sufridos por los conductores de ómnibus. Lima, Perú

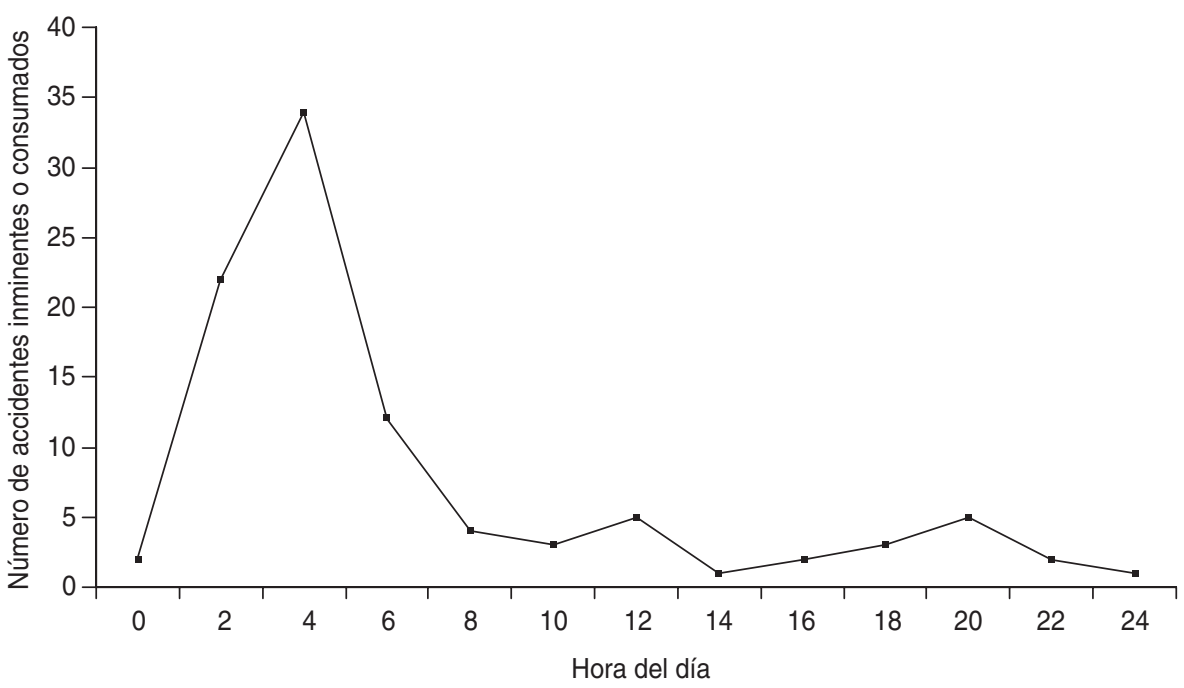

durante la conducción (55\%), imprudencia del chofer $(24 \%)$, exceso de velocidad (5\%), ebriedad (4\%), falla mecánica $(3 \%)$, mal estado de las pistas (1\%) y negligencia de las empresas de transportes (1\%). De otro lado, cuando se preguntó sobre la causa del accidente o del suceso que estuvo a punto de serlo, las respuestas fueron: imprudencia del otro chofer implicado $(49 \%)$, cansancio (19\%), falla mecánica $(11 \%)$, inclemencias del tiempo (8\%), imprudencia del chofer encuestado (4\%), imprudencia de peatones $(3 \%)$ y mal estado de las pistas (2\%). Del total de conductores encuestados, $71 \%$ afirmaron tener noticia de compañeros que habían sufrido accidentes de carretera a causa de la somnolencia.

Admitieron haberse accidentado o haber estado a punto de accidentarse (grupo A) $45 \%$ de los encuestados, mientras que los demás (55\%) negaron cualquier incidente de este tipo (grupo B). El análisis bifactorial no reveló la asociación de dicho antecedente con el informe de ronquido o pausas respiratorias durante el sueño $(P=0,81$ y 0,22 , respectivamente). Sin embargo, el pestañeo y el cansancio durante la conducción mostraron una firme relación con dicho antecedente $(P<0,0005)$. No se encontró ninguna asociación de los años de experiencia del chofer, número de horas de conducción al día o índice de masa corporal (IMC), con el dato de haberse accidentado o casi accidentado ( $P=0,14,0,34$ y 0,21 respectivamente).

Recordaron la hora en que estuvieron a punto de tener un accidente o lo tuvieron $99 \%$ de los conductores y, como puede apreciarse en el histograma de frecuencias de la figura 1 , se distinguen tres picos; el más definido entre las 00.00 y 06.00 horas, y los otros dos entre las 12.00 y 14.00 y entre las 20.00 y 22.00 horas.

\section{DISCUSIÓN}

La tasa de reclutamiento fue adecuada, con niveles bajos de rechazo, lo que permite considerar que la muestra es representativa de la población general de conductores de ómnibus que laboran en esta importante terminal terrestre de Lima, Perú. Los conductores encuestados fueron exclusivamente varones, de una edad promedio de 42,5 años (límites: 19 y 73 años). y con un índice de masa corporal (IMC) promedio elevado. Al agruparlos por horas de sueño al día, se observó que una proporción importante de los choferes duerme menos de 7 y de 6 horas diarias, que sería la cantidad aceptable para la edad de este grupo. No obstante, la información a este respecto es más relevante cuando se pregunta por el tiempo de sueño en las últimas 24 horas; planteado así, la tercera parte de los encuestados declaró haber dormido menos de 6 horas. Esta información indica que una proporción importante de los conductores incluidos en el estudio experimenta privación aguda y crónica del sueño.

Cerca de $50 \%$ de los sujetos conducen sus vehículos más de 5 horas seguidas, y el cansancio durante la conducción se presenta fundamentalmente en las madrugadas y las tardes. Los encuestados no respetan el período de conducción recomendado por la normativa vigente del Ministerio de Transportes y Comunicaciones, que limita la actividad a 4 horas (13). La tercera parte de los conductores reconocieron haber experimentado pestañeo, que es un indicio extremo de somnolencia al volante. La alta frecuencia de cansancio y somnolencia patológica constituye un dato preocupante en esta serie y ubica a los conductores de ómnibus de la Carretera Panamericana Norte del Perú como elemento de alto riesgo en la causalidad de accidentes.

Cerca de $80 \%$ de la población encuestada duerme en el compartimiento destinado para el equipaje. Lo más llamativo es que casi todos lo hacen cuando la unidad está en movimiento, recorriendo la ruta. La llamada bodega o maletero resulta menos que impropia para ese fin, ya que constituye un espacio estrecho, que carece de ventilación adecuada, no abre sino desde el exterior y no ofrece protección al chofer en eventuales colisiones. Resulta evidente que este hábito generalizado entre los conductores resulta, además de peligroso para su integridad física, también incómodo y totalmente opuesto a lo que sería un ambiente apropiado para lograr un sueño de buena calidad.

La clasificación internacional de trastornos del sueño define las alteraciones de origen ambiental —que son parte de las llamadas disomnias- en términos de insomnio y somnolencia excesiva (1). Al parecer, esta costumbre, arraigada en los conductores de la Carretera Panamericana Norte, no 
tiene precedente en la literatura médico-laboral que aborda el tema. De otro lado, dormir en el maletero convierte en letra muerta la norma vigente del Ministerio de Transportes y Comunicaciones del Perú, que prohíbe expresamente utilizar el compartimiento para ese fin (13).

$\mathrm{Al}$ parecer convencidos de lograr su objetivo, los conductores recurren a una variedad de maniobras en su afán por evitar dormirse al volante. Escuchar música, abrir la ventana lateral para refrescarse, fumar y beber café son maniobras empleadas por estos conductores, como lo son por los de otras latitudes $(14,15)$. En particular, las dos primeras no disminuyen la somnolencia, según concluyen estudios de sujetos sometidos a privación de sueño y evaluados en condiciones de laboratorio con equipos de conducción simulada y monitor electroencefalográfico (16). De acuerdo con esos autores, dichas prácticas confieren un beneficio transitorio y marginal, que en el mejor de los casos brindaría al conductor un lapso apenas suficiente para buscar un lugar adecuado donde descansar.

No se ha encontrado información relacionada con hábitos locales, tales como mojarse — que es, por cierto, otra maniobra inútil para controlar la somnolencia- o beber una mezcla de alcohol con hoja de coca. Sin embargo, se sabe que el alcohol, aun en cantidades pequeñas, produce deterioro de las destrezas cognoscitivas y motoras en conductores con privación aguda del sueño (17). Por otro lado, se ha demostrado que un período de 17 horas de restricción del sueño ocasiona en esas funciones un deterioro similar al que causaría una alcoholemia del orden de $0,05 \%$, y que tras una privación de sueño de 24 horas el déficit cognoscitivo-psicomotor equivale al que produciría una concentración de etanol en sangre del orden de $0,10 \%$ (18-19). Esos datos demuestran que conducir vehículos en condiciones de privación del sueño es tan peligroso como hacerlo con niveles proscritos de alcoholemia.

En cuanto a la cafeína, una dosis de $150 \mathrm{mg}$ logra disminuir notablemente, durante dos horas, la somnolencia, la frecuencia del parpadeo y el trazado electroencefalográfico de somnolencia en personas jóvenes y sanas privadas parcialmente de sueño la noche anterior (15). En sujetos que han dormido cinco horas la noche anterior, una dosis de $200 \mathrm{mg}$ de cafeína (equivalente a dos o tres tazas de café) y el dormir una siesta reducen por períodos de dos horas y media hora, respectivamente, el deterioro cognoscitivo y la percepción subjetiva de somnolencia durante la conducción. En cambio, ese nivel de "protección", con la misma dosis de cafeína, se pierde cuando el sujeto no duerme la víspera, ya que la somnolencia y parámetros similares disminuyen por un lapso de apenas 30 minutos (20). Según demostraron los estudios originales de Horne et al. (21) y Cummings et al. (22), los conductores pueden reducir el riesgo de accidentes de carretera si detienen el vehículo al percibir cansancio o somnolencia, eligen lugares adecuados para descansar a lo largo de su recorrido y evitan conducir largas distancias sin períodos intermedios de reposo.

La terminal Fiori no ofrece ambientes acondicionados para el reposo de los trabajadores y son excepcionales las empresas de transporte que facilitan el descanso de sus conductores en hoteles a lo largo de la ruta. Aunque no ha sido objetivo primario del estudio, la encuesta pone de manifiesto las deplorables condiciones laborales de los conductores encuestados, la indiferencia de los empresarios del transporte de pasajeros por carretera y la indolencia de las autoridades responsables ante la violación sistemática de la normativa vigente. Hacen lo suyo también el entorno informal y la irregularidad de los horarios de trabajo. En las condiciones actuales de crisis económica y desempleo, estos elementos vienen a tener un peso muy importante en la génesis del problema que aquí se trata.

El ronquido estuvo presente en proporciones similares a lo informado en otros grupos poblacionales encuestados en nuestro medio (23-25). Sin embargo, en el presente análisis la somnolencia mostró una frecuencia más alta, lo que puede explicarse por la acumu- lación de turnos a causa de la rotación anómala de los horarios de trabajo (actividades diurnas y nocturnas), la privación aguda y crónica del sueño y trastornos del ambiente, a juzgar por la información obtenida en este estudio. Las encuestas antes mencionadas corresponden a poblaciones sin alteración del ritmo circadiano.

El cansancio y la somnolencia en conductores de la terminal Fiori son inespecíficos y no necesariamente se vinculan con trastornos orgánicos como el síndrome de apnea-hipopnea del sueño (SAHS), síndrome de piernas inquietas (SPI), movimiento periódico de miembros (MPM) o narcolepsia, por mencionar algunos. Sin embargo, ante las cifras de prevalencia que ofrecen los informes epidemiológicos de Young et al. (26) y Durán et al. (27), no es del todo impensable que algunos conductores padezcan SAHS, si bien el presente estudio no permite extraer conclusiones al respecto, porque la evaluación de tales variables no fue uno de sus objetivos.

La alternancia sueño-vigilia está enmarcada dentro del concepto de la denominada regulación circadiana del sueño (28). En condiciones normales el marcapaso interno define las horas en que el sujeto debe dormir y aquellas en que permanece despierto. Las exigencias de la vida moderna y la industrialización han trastocado en buena medida el ritmo natural del ciclo circadiano en el ser humano de nuestra época, como bien lo ilustra el caso presente de los conductores de ómnibus. Las condiciones laborales les exigen trabajar durante las tardes, noches y aun en las madrugadas, períodos del día en que la presión funcional por dormir es mucho más intensa, de acuerdo con ese patrón natural de alternancia. Así, ya que conducir durante la tarde o la madrugada es por sí mismo peligroso, no es de extrañar que el consecuente cansancio de los conductores incremente el riesgo de accidentes de tránsito a esas horas, como señala la literatura médica $(29,30)$. El histograma de frecuencias refleja claramente la marcada tendencia de los conductores encuestados a sufrir o a casi sufrir accidentes fundamentalmente en horas de la madrugada, en 
concordancia con las publicaciones, por ejemplo, del Panel conjunto sobre fatiga y somnolencia en los conductores del National Center for Sleep Disorders Research y la National Highway Traffic Safety Administration (NCSDR/ NHTSA) de Estados Unidos (2), además de los estudios de Lyznicki et al. (3), Horne y Reyner (4) y Powell et al. (31).

De acuerdo con el Ministerio de Transportes y Comunicaciones del Perú, en el año 2000 se produjeron en el país 33063 accidentes de tránsito, que dieron un saldo de 29995 heridos y 3118 muertos (32). Esta institución estatal señala que $10 \%$ de los accidentes fueron provocados por ómnibus y que la tasa de accidentes por tipo de vehículo causante fue de 32 por cada 100 ómnibus y de 9,7 por cada 100 automóviles que circularon durante el año 1999 en territorio peruano (33). Por otro lado, el Patronato de Seguridad Vial afirmó que 3400 personas fallecieron en las autopistas del Perú en 1995, a razón de 40 muertos por cada 10000 automóviles, tasa abrumadoramente más alta que las registradas en Estados Unidos o en Suecia, que apenas llegan a 2 por cada 100000 (34). Si bien estas variables no atañen directamente al tema central del presente informe, parece pertinente mencionarlas, ya que esas cifras impresionantemente distintas ponen de manifiesto la altísima siniestralidad que se observa en el Perú. La Dirección Nacional de Policía de Carreteras registró 389 y 527 accidentes de ómnibus en carreteras durante los años 1999 y 2000, respectivamente; el número de heridos / muertos fue 2 074/277 y 2 506/275, respectivamente (35). Estas cifras indican una alta tasa de siniestralidad, aunada a un alto número de heridos y muertos por accidente. Resulta particularmente preocupante la elevada tasa de accidentes causados por ómnibus, unidades de transporte que usualmente llevan entre 45 y 52 pasajeros.

¿Cuáles son las causas de los accidentes de ómnibus en el Perú? Según la Dirección General de Circulación Terrestre, las principales causas de accidente que implicaron a empresas de transporte de pasajeros durante el año 2000 fueron: imprudencia del conduc- tor, $28 \%$; invasión del carril opuesto, $16 \%$; mal tiempo, $15 \%$; exceso de velocidad, 12\%; imprudencia del peatón, $10 \%$ y ebriedad del conductor, $4 \%$ (32). Mientras que en esta fuente de consulta el cansancio y la somnolencia no figuran como causas de accidentes, los resultados del presente estudio indican todo lo contrario. Los protagonistas centrales del informe opinaron que el cansancio es la primera causa de accidentes de ómnibus en las carreteras del Perú y la segunda que originó un accidente o lo que pudo haber sido un accidente en la experiencia personal del encuestado. Igualmente, las tres cuartas partes de la población de estudio sabían de compañeros que se accidentaron por cansancio. Como puede observarse, se emplearon ex profeso tres formas de preguntar sobre una misma variable con el fin de obtener información más concreta. La pregunta relacionada con el antecedente del propio conductor de haberse accidentado o casi accidentado es la más relevante. Por ello parece difícil sustentar que los accidentes de ómnibus en la carretera Panamericana Norte no sean generados, entre otras causas, por cansancio $\mathrm{y}$ somnolencia.

¿Por qué la información estadística oficial no es congruente de alguna manera con nuestros hallazgos? Esto podría explicarse ante todo por los métodos de recolección de información y el razonamiento pericial en el lugar de los hechos. Si la variable "accidente por somnolencia o cansancio" no se registra en la ficha del informe policial ni se toma en cuenta como hipótesis por el perito, difícilmente aparecerá en el consolidado final y probablemente la tendencia sea calificarla como "imprudencia del conductor", ya que el investigador atribuirá la responsabilidad directa al chofer involucrado. Así, esta variable se convierte en un cajón de sastre donde queda registrada una buena proporción de los accidentes. En segundo lugar, es probable que el personal responsable no esté capacitado para identificar el siniestro como tal y por ello no lo califique adecuadamente.

Se encontró una relación entre el cansancio y el pestañeo durante la conducción y el antecedente de haberse ac- cidentado o casi accidentado. Este dato apoya la hipótesis de que hay una relación entre la somnolencia patológica durante la conducción y los accidentes de tránsito en las carreteras. Sin embargo, el diseño del estudio no permite establecer la dirección de la relación causa-efecto. Por otro lado, preocupa el haber hallado privación aguda y crónica del sueño, trastorno del ambiente durante el sueño, rotación de horarios de trabajo de los conductores y una alta frecuencia de accidente inminente o consumado durante la madrugada.

El estudio tiene las limitaciones inherentes a la recopilación de información por medio de un cuestionario. El sesgo de recordación y consideraciones subjetivas podrían influir en las respuestas sobre el número de horas de sueño o el de horas de permanencia al volante sin descansar. Los valores proporcionados por los conductores representan cifras subjetivas relacionadas con el ciclo sueño-vigilia, mientras que el método ideal de abordar estas variables es la cuantificación objetiva con actímetros (36). Razones de costo impidieron utilizar estos aparatos, así que se optó por el sistema de encuesta supervisada como instrumento principal de recopilación. A pesar de estas limitaciones, los resultados tienen particular relevancia y abren el camino para futuras investigaciones sobre los accidentes en carreteras y su relación con la somnolencia. Se ha procurado precisión en la presentación de los resultados y cautela en el análisis de los que implican el uso de los términos "cansancio" o "somnolencia", de acuerdo con la manera como se formularon las preguntas en el cuestionario. Conviene recalcar que, según el estudio cualitativo y la prueba piloto del cuestionario, los términos "somnolencia", "tener sueño" o "dormirse" durante la conducción de las unidades no forman parte del vocabulario de los conductores de ómnibus que participaron en el estudio. Aunque la afirmación requiere una evaluación más puntual, tenemos la impresión de que la mención explícita de estas palabras implicaría, para los conductores, la aceptación de responsabilidad directa en la génesis de los accidentes. La diferenciación de los términos "can- 
sancio" y "somnolencia" es objetiva, lo cual permite presentar los resultados con rigor y evitar que aquellos sean interpretados como sinónimos, ya que no lo son en realidad. Como se comentó antes, el diseño del estudio no determina la dirección de la relación entre somnolencia-cansancio y los accidentes en las carreteras, si bien lo más lógico y coherente es pensar que primero ocurre el cansancio o la somnolencia y luego el accidente, y no a la inversa.

\section{CONCLUSIONES}

Los resultados demuestran que, en la población evaluada, el cansancio y la somnolencia durante la conducción son frecuentes y que tienen causas multifactoriales secundarias a la privación aguda y crónica del sueño, la rotación de los horarios de trabajo, trastornos del ambiente y, probablemente, trastornos primarios del sueño. Los conductores de ómnibus no siguen una sistemática de trabajo coherente con el ciclo sueño-vigilia — conducen indistintamente de día, noche o madrugada-, duermen en condiciones ambientales deplorables, y emplean, para evitar la somnolencia durante la conducción, una variedad de maniobras probadamente ineficaces del todo para estos fines. La informalidad y el incumplimiento de las normas establecidas son la regla, más que la excepción. También fue frecuente la experiencia de tener un accidente o estar a punto de tenerlo y llamó la atención su elevada frecuencia en horas de madrugada, hecho que respalda la hipótesis de su relación con el cansancio y la somnolencia. Los entrevistados afirmaron que por lo menos $20 \%$ de esas experiencias funestas fueron provocadas por cansancio durante la conducción y, en su opinión, el cansancio es la principal causa de accidentes de carretera en el Perú.

Es necesario ahondar en el tema aquí planteado mediante nuevas investigaciones que incluyan a conductores de ómnibus, de camiones y de los otros medios de transporte motorizados que circulan por las distintas carreteras y zonas urbanas del país. Parece urgente elaborar normas más precisas en cuanto a los horarios de trabajo de los conductores, aplicarlas de manera eficaz y vigilar con rigor su cumplimiento. Debe ge- neralizarse el uso de taquímetros para controlar el tiempo de conducción y de alternancia de los conductores. Es necesario establecer centros de reposo para choferes a lo largo de las carreteras, a fin de que su actividad laboral pueda programarse de una manera sistemática, más acorde al ciclo natural de sueñovigilia. Es también indispensable llevar a cabo intervenciones educativas y realizar un trabajo integrado con la participación de todas las entidades relacionadas con el problema, como son los legisladores, las autoridades policiales, los transportistas, los conductores y los especialistas en medicina laboral. Los formatos periciales de registro de accidentes en carreteras deberían incluir, como elementos de registro, el cansancio y el número de horas de sueño en las 24 horas previas. Los resultados aquí presentados son alarmantes e indican que el cansancio y la somnolencia durante la conducción, como causas de accidentes, merecen especial atención como problema de salud pública. La siniestralidad no cambiará mientras no se instauren medidas eficaces dirigidas hacia un fin específico: los conductores deben dormir bien.

\section{REFERENCIAS}

1. American Sleep Disorders Association. The International Classification of Sleep Disorders: Revised Diagnostic and Coding Manual. Rochester (MN): ASDA; 1997. P. 77.

2. Expert Panel on Driver Fatigue and Sleepiness (NCSDR/NHTSA). Drowsy driving and automobile crashes. Washington, D.C.: National Highway Safety Association; 1998 (Dot HS 808 707).

3. Lyznicki JM, Doege TC, Davis RM, Williams MA. Sleepiness, driving, and motor vehicle crashes. JAMA. 1998;279: 1908-13.

4. Horne JA, Reyner LA. Sleep-related vehicle accidents. Br Med J. 1995;310(6979): 565-67.

5. Garbarino S, Nobili L, Beelke M, De Carli F, Ferrillo F. The contributing role of sleepiness in highway vehicle accidents. Sleep. 2001;24(2):203-6.

6. Akerstedt T. Consensus statement: Fatigue and accidents in transport operations. J Sleep Res. 2000;9:395.
7. Rey de Castro J. Accidentes de tránsito en carreteras e hipersomnia durante la conducción: la evidencia periodística. Rev Med Her. 2003;14:69-73.

8. Rey de Castro J, Soriano S. Hipersomnia durante la conducción de vehículos: ¿causa de accidentes en carreteras? A propósito de un estudio cualitativo. Rev Soc Peru Med Intern. 2002;15:142-9.

9. McCartt AT, Rohrbaugh JW, Hammer MC, Fuller S. Factors associated with falling asleep at the wheel among longdistance truck drivers. Accid Anal Prev. 2000;32:493-504.

10. Johnson EO. Sleep in America: 1999. Results from the National Sleep Foundation's 1999 Omnibus Sleep Poll. Washington, D.C.: National Sleep Foundation; 1999. Pp. 42-9. [Citado el 24 mayo de 2004]. Hallado en: http:/ / www.sleepfoundation. org/publications/1999poll.cfm

11. WB\&A Market Research. 2002 Sleep in America Poll. Washington, D.C.: National Sleep Foundation; 2002. P. 25. [Citado el
24 de mayo de 2004]. Hallado en: http:/ / www.sleepfoundation.org/img/2002 sleepinamericapoll.pdf

12. Rey de Castro J, Vizcarra D. Síndrome apnea sueño obstructiva: descripción clínica y polisomnográfica en 48 pacientes. Bol Soc Peru Med Intern. 1999;12:2-7.

13. Reglamento Nacional de Administración de Transportes DS-040-2001-MTC. En: De los conductores, Prohibiciones, Art. $301 \mathrm{~h}$ y 303. Diario El Peruano, Normas Legales, 28 de julio de 2001. P. 207863.

14. Maycock G. Slepiness and driving: the experience of UK car drivers. J Sleep Res. 1996;5:229-37.

15. Horne JA, Reyner LA. Counteracting driver sleepiness: effects of napping, caffeine and placebo. Psychophysiology. 1996;33: 306-9.

16. Reyner LA, Horne JA. Evaluation on "Incar" countermeasures to sleepiness: cold air and radio. Sleep. 1998;21:46-50.

17. Arnedt JT, Wilde GJS, Munt PW, MacLean AW. Simulated driving performance 
following prolonged wakefulness and alcohol consumption: separate and combined contributions to impairment. J Sleep Res. 2000;9:233-41.

18. Dawson D, Reid K. Fatigue, alcohol and performance impairment. Nature. 1997; 388:235.

19. Williamson AM, Feyer AM. Moderate sleep deprivation produces impairment in cognitive and motor performance equivalent to legally proscribed levels of alcohol intoxication. Occup Environ Med. 2000;57:649-55.

20. Reyner LA, Horne JA. Early morning driver sleepiness: effectiveness of $200 \mathrm{mg}$ caffeine. Psycophysiology. 2000;37:251-6.

21. Horne JA, Reyner LA. Driver sleepiness. J Sleep Res. 1995;4 Suppl 2:23-9.

22. Cummings $P$, Koespsell TD, Moffat JM, Rivara FP. Drowsiness, counter-measures to drowsiness, and the risk of motor vehicle crash. Injury Prev. 2001;7:194-9.

23. Rey de Castro J, Hernández J. Síntomas relacionados al síndrome apnea-hipopnea obstructiva del sueño. ¿Son frecuentes en el consultorio de neumología? Bol Soc Peru Med Int. 2000;13:196-200.

24. Rey de Castro J, Vizcarra D. Frecuencia de síntomas del síndrome de apnea-hipopnea del sueño e insomnio en médicos de una clínica privada peruana. Rev Med Hered. 2003;14:53-8.

25. Pacheco G, Rey de Castro J. Insomnio en pacientes adultos ambulatorios de me- dicina interna del Hospital Arzobispo Loayza. Rev Med Hered. 2003;14:63-8.

26. Young T, Palta M, Dempsey J, Skatrud J, Weber S, Bard S. The occurrence of sleep disorders breathing among middle-aged adults. N Engl J Med. 1993;328:1230-6.

27. Durán J, Esnaola S, Rubio R, Iztueta A. Obstructive sleep apnea-hipopnea and related clinical features in a populationbased sample of subjects aged 30 to 70 years. Am J Respir Crit Care Med. 2001; 163:685-9.

28. Kilduff T, Kushida CA. Circadian regulation of sleep. En: Chokroverty S, ed. Sleep disorders medicine: basic science, technical considerations, and clinical aspects. Burlington (MA): Butterworth Heinemann; 1999. P. 135.

29. Philip P, Taillard J. Dette de somneil et risqué accidents chez les jeunes conducteurs. En: Léger D, Guilleminault C, eds. Somneil, vigilance et travail. Paris: Masson; 1997. P. 149.

30. Philip P, Ghorayeb I, Menny JC, DemontesMainard J, Gien M, Guilleminault C, et al. Determinants of sleepiness in automobile drivers. Sleep Res. 1995;24:108.

31. Powell NB, Schechtman KB, Riley RW, Li $\mathrm{K}$, Troell R, Guilleminault C. The road to danger: the comparative risks of driving while sleepy. Laryngoscope. 2001;111: 887-93.

32. Ministerio de Comunicaciones y Transportes, Gobierno del Perú. Víctimas por accidentes de tránsito nacional, según características: 1990-2000. Cuadro $\mathrm{N}^{\circ}$ 3.G.4 y 3.E.2. [Citado el 24 de mayo 2004] Hallado en: http://www.mtc.gob.pe/ estadisticas/archivos/xls/3.G.4.xls

33. Oficina General de Métodos y Sistemas. Dirección de Estadística, Gobierno del Perú. Accidentes de Tránsito 1995-1999. Boletín del Ministerio de Transportes, Comunicaciones, Vivienda y Construcción, Diciembre 2000, Lima-Perú.

34. Cruzada vial: Patronato de seguridad vial se echa a rodar. Revista Caretas. 1996 sep 26; secc. Mar de Fondo. Lima, Perú. [Citado el 24 de mayo 2004]. Hallado en: http://www.caretas.com.pe/1433/mdf/ mdf.htm

35. Registro Estadístico de la Dirección Nacional Policía de Carreteras DINPOLCAR-PNP OFATEC. Años 1999 y 2000. Lima: Ministerio del Interior.

36. Ancoli-Israel S, Cole R, Alessi C, Chambers $\mathrm{M}$, Moorcroft W, Pollack CP. The role of actigraphy in the study of sleep and circadian rhythms. Sleep 2003;26:342-92.

Manuscrito recibido el 26 de septiembre de 2003. Aceptado para publicación, tras revisión, el 6 de mayo de 2004.
ABSTRACT

\section{Tiredness and sleepiness in bus drivers and road accidents in Peru: a quantitative study}

Objective. To evaluate the relationship that tiredness and sleepiness in bus drivers have to road accidents in Peru. Information from various countries indicates that driver sleepiness plays an important role in road accidents. However, there is only limited information on this subject in Peru.

Methods. Using a supervised, pretested survey, a cross-sectional observational and comparative study was carried out with 238 bus drivers who drive on the Northern Pan American Highway of Peru. To determine the relationship between variables the chisquare test was used, along with the Pearson correlation coefficient. The level of significance was set at $P<0.05$. The variables analyzed were: tiredness, sleepiness, hours of driving per day, daily hours of sleep, body mass index, snoring, sleep apnea, and either having had or almost having had an accident while driving.

Results. Of the 238 drivers, all of them were men, $45 \%$ said they had had or nearly had had an accident while driving, 55\% slept less than 6 hours per day, 31\% had slept less than 6 hours in the 24 hours before answering the survey, and $80 \%$ were in the habit of driving more than 5 hours without stopping. Of the drivers, $56 \%$ of them reported being tired at least some of the time while driving; of this group, $65 \%$ of them reported being tired during the early morning. Seventy-six drivers (32\%) said that while they were driving their eyes had fallen shut. In terms of where they slept, 194 of the drivers $(81 \%)$ said they always slept in the lower luggage compartment of the bus while another driver was driving the bus or when the bus was parked in the bus terminal. The steps that drivers took to avoid falling asleep while driving included: wetting the face with water, eating fruit, opening the window of the driver's compartment, drinking coffee, listening to music, smoking, chewing coca leaves, and drinking alcohol mixed with coca leaves. In the opinion of $55 \%$ of the drivers, the leading cause of road accidents is tiredness. Accidents and near-accidents while driving occurred mainly between midnight and 6 a.m. Having an accident or a near-accident was strongly associated with tiredness and with having the eyes drop shut while driving $(P<0.0005)$.

Conclusions. Tiredness and sleepiness while driving were common among the bus drivers, with various possible causes: acute and chronic sleep deprivation, irregular schedule changes, and sleep disorders due to the drivers' working conditions. Our results support the hypothesis that fatigue and sleepiness among bus drivers are related to road accidents. 